\title{
A Case of Angioimmunoblastic T-cell Lymphoma Hidden in Plain Sight: A Delay in Diagnosis and Management
}

\author{
Ndausung Udongwo ${ }^{\mathrm{a}, \mathrm{e}}$, Nusha Fareen ${ }^{\mathrm{a}}$, Hayley Fried ${ }^{\mathrm{b}}$, Jennifer Nessim ${ }^{\mathrm{a}}$, Lakshmi Rehka Narra ${ }^{\mathrm{a}}$, \\ Dhairya Gor ${ }^{\text {a }}$, Varsha Gupta ${ }^{\mathrm{c}}$, Narmeen Farooq ${ }^{\mathrm{d}}$, Saira Chaughtai ${ }^{\mathrm{a}}$
}

\begin{abstract}
Angioimmunoblastic T-cell lymphoma (AITL) is an uncommon type of cluster of differentiation (CD)4 T-cell peripheral lymphoma. The varied clinical presentations of AITL present a challenge for accurate diagnosis. We present a case of a 57-year-old female with a history of severe acute respiratory syndrome coronavirus 2 (SARS-CoV-2) infection in May 2020, who presented to the hospital in the summer of 2021 with shortness of breath for 3 months. She underwent an extensive workup for lymphadenopathy while in Canada involving multiple core lymph node biopsies, which were inconclusive. Here in our institution, several tests for infectious diseases were unremarkable. Imaging tests revealed bilateral pleural effusion, lymphadenopathies, and rectal thickening. Results from rectal biopsy and excisional cervical lymph node biopsy revealed findings typical of AITL. Due to worsening hypoxia with pleural fluid accumulation, bilateral chest tubes (PleurX catheter) were placed. Steroids and chemotherapy were started. She was discharged in stable condition to follow-up care. An integrated and persistent approach comprising clinical, morphologic, excisional biopsy, immunophenotyping, and molecular tests is essential in reaching a correct diagnosis of AITL. Through our consistent effort to obtain further imaging and tissue biopsies, we came to the diagnosis which allowed her to begin appropriate life-saving treatments.
\end{abstract}

Keywords: Angioimmunoblastic T-cell lymphoma; Excisional biopsy; Immunophenotyping; Lymphadenopathy; Lymph nodes; NonHodgkin lymphoma; Chemotherapy; Core needle biopsy

\section{Introduction}

Angioimmunoblastic T-cell lymphoma (AITL) is an uncom-

Manuscript submitted December 29, 2021, accepted February 5, 2022

Published online March 5, 2022

aDepartment of Internal Medicine, Jersey Shore University Medical Center, Neptune City, NJ 07753, USA

bRowan School of Medicine, Stratford, NJ 08084, USA

'Department of Hematology and Oncology, Jersey Shore University Medical Center, Neptune City, NJ 07753, USA

${ }^{d}$ Niazi Medical and Dental College, Sargodha, Pakistan

${ }^{e}$ Corresponding Author: Ndausung Udongwo, Department of Internal Medicine, Jersey Shore University Medical Center, Neptune City, NJ 077543, USA.

Email: ndausung.udongwo@hmhn.org

doi: https://doi.org/10.14740/jmc3890 mon type of cluster of differentiation (CD) $4 \mathrm{~T}$ cell peripheral lymphoma [1]. It has an incidence of $0.00005 \%$ in the USA. It is more prevalent in men compared to women, and usually occurs between the ages of $60-70[1,2]$. AITL has a median survival of $<3$ years [1]. Excisional lymph node biopsy with concomitant immunophenotypic, molecular, and cytogenetic studies are required for an accurate diagnosis of this disease [1]. We present a case of a 57-year-old female with a delayed diagnosis of AITL.

\section{Case Report}

\section{Investigations}

A 57-year-old female with a history of severe acute respiratory syndrome coronavirus 2 (SARS-CoV-2) infection in May 2020 , presented to the hospital in the summer of 2021 with shortness of breath for about 3 months but got worse 3 days before coming to the emergency department. It was associated with fatigue, dry cough, vomiting, and non-bloody diarrhea. She denied any fever or weight loss. The patient was found with lymphadenopathy involving the neck in the early part of 2021, she underwent an extensive workup for lymphadenopathy, while in Canada, where she originally resides. She underwent multiple biopsies including fine-needle aspiration of the posterior cervical lymph nodes and core biopsies from axillary lymph nodes that were inconclusive. Of note, she never received coronavirus disease 2019 (COVID-19) vaccines. On initial assessments, vitals were that blood pressure was $110 / 80$ $\mathrm{mm} \mathrm{Hg}$; heart rate was 87 beats per minute; respiratory rate was 12 breaths per minute; temperature was $36.6^{\circ} \mathrm{C}$; oxygen saturation was $85 \%$ that improved to $96 \%$ on 2 -L nasal cannula. On physical examination, there were bilateral palpable tender submental, submandibular, posterior auricular, cervi$\mathrm{cal}$, supraclavicular, axillary, and inguinal lymph nodes. There were decreased breath sounds bilaterally on the lower lung fields with fine crackles (rales). The patient was admitted for management of acute hypoxic respiratory failure with suspicion for undiagnosed infection or neoplasm.

\section{Diagnosis}

Laboratory tests showed hemoglobin of $10.3 \mathrm{~g} / \mathrm{dL}$ (normal 
Table 1. Laboratory Studies Done During Admission

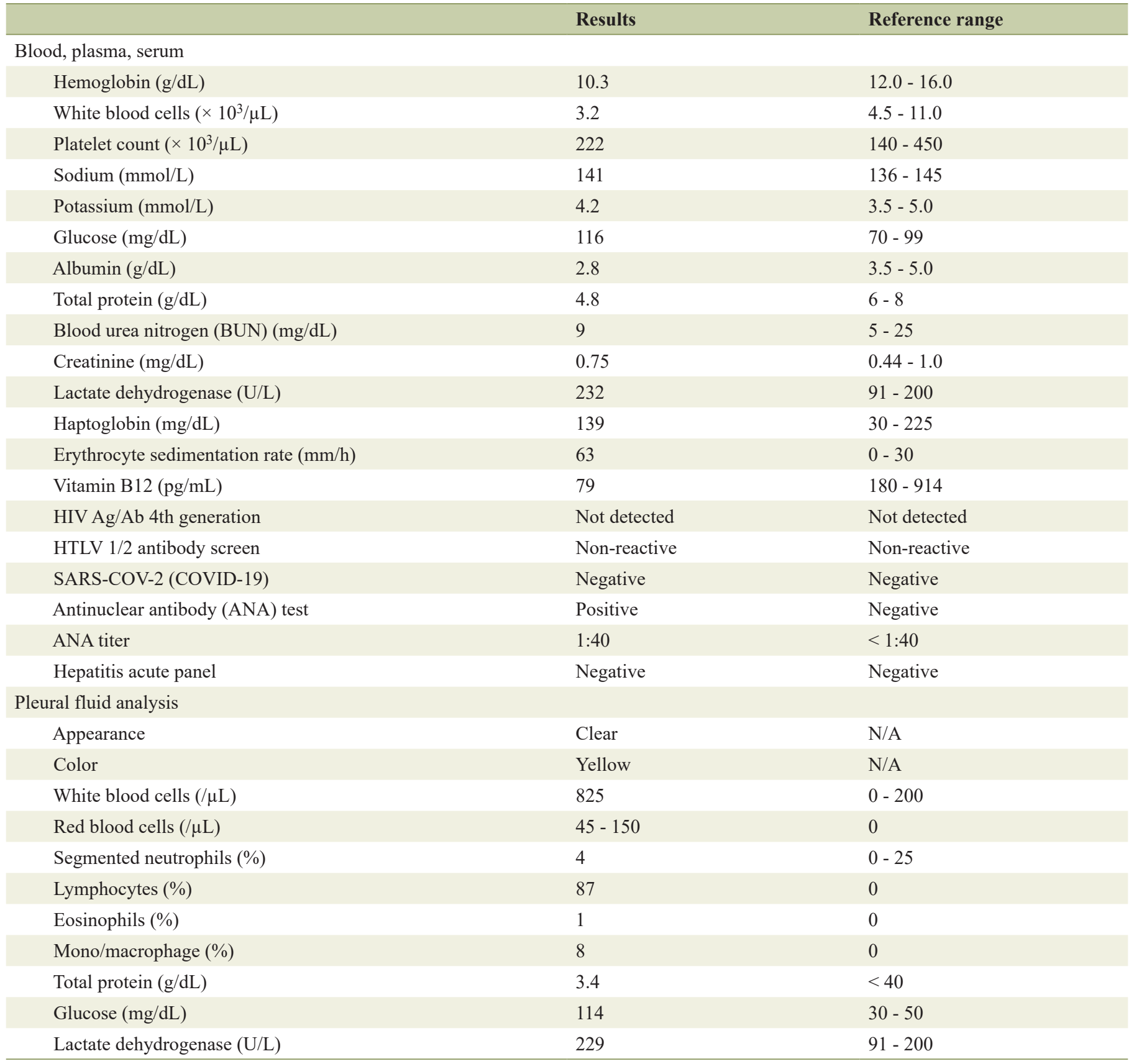

HIV: human immunodeficiency virus; HTLV: human T-lymphotropic virus; COVID-19: coronavirus disease 2019; SARS-CoV-2: severe acute respiratory syndrome coronavirus 2.

range: 12 - $16 \mathrm{~g} / \mathrm{dL}$ ), leukocyte count of $3.2 \times 10^{3} / \mu \mathrm{L}$ (normal range: $\left.4.5-11 \times 10^{3} / \mu \mathrm{L}\right)$, erythrocyte sedimentation rate of $63 \mathrm{~mm} / \mathrm{h}$ (normal range: $0-30 \mathrm{~mm} / \mathrm{h}$ ), and antineutrophil antibody titer of 1:40 (normal range: $<1: 40$ ) as shown in Table 1. Also, SARS-CoV-2, human immunodeficiency virus, Epstein-Barr virus (EBV), hepatitis panel, and tuberculosis tests were negative (Table 1). A computed tomography scan of the chest/abdomen without contrast revealed prominent bilateral pleural effusions, right lower paratracheal, pre-carinal lym- phadenopathy, paraoesophageal lymphadenopathy with multiple bilateral axillary lymph nodes, and rectal wall thickening (Fig. 1a-f). Bilateral thoracentesis drained 1,200/1,400 mL of fluid in the left/right pleural cavity, respectively. Pleural fluid analysis showed lymphocyte predominance with negative cytology, while light's criteria revealed an exudative pleural effusion (Table 1). Colonoscopy with rectal biopsy revealed results consistent with peripheral T-cell lymphoma (Fig. 2a, b). Excisional cervical lymph node biopsy revealed architec- 

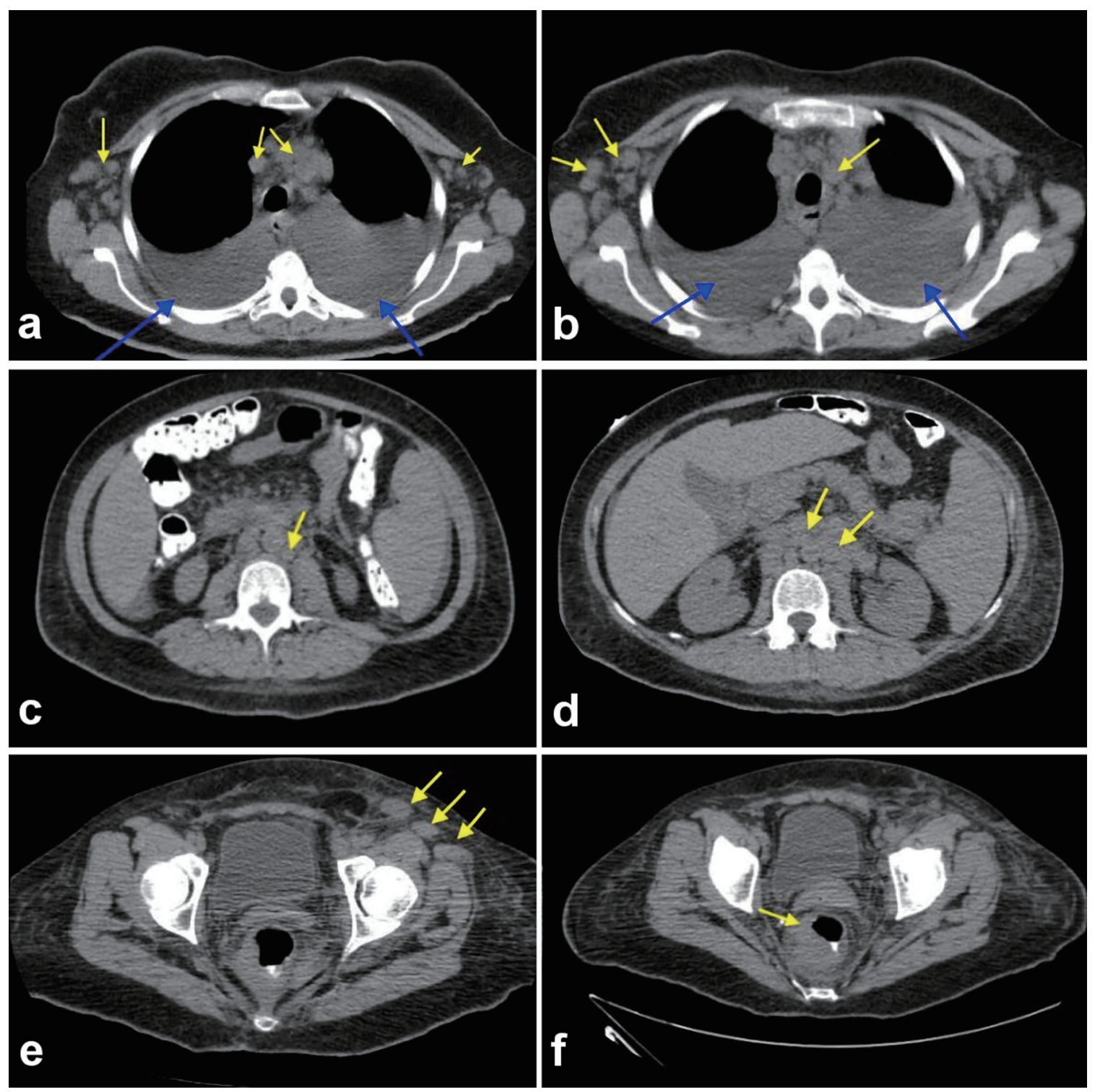

Figure 1. (a-f) Computed tomography scan of chest, abdomen, and pelvis showing an extensive lymphadenopathy in the bilateral axillary region and in the mediastinum (yellow arrows) with bilateral pleural effusion noted (blue arrows). (a) Extensive lymphadenopathy in bilateral axillary region and in the mediastinum (yellow arrows). (b) Extensive lymphadenopathy involving the bilateral thoracic region and mediastinum (yellow arrows). (c) Extensive paraaortic and pre-vertebral lymphadenopathy (yellow arrows). (d) Extensive paraaortic wall lymphadenopathy (yellow arrows). (e) Extensive pelvic wall lymphadenopathy. (f) Discrete mass surrounding the rectum suspicious for malignancy.

ture typical of AITL. Immunophenotyping and morphological characteristics of the neoplastic cells confirmed the diagnosis (Fig. 3a-e).

\section{Treatment}

Due to worsening hypoxia with pleural fluid accumulation, bilateral chest tubes (PleurX catheter) were placed. Once AITL was confirmed, she was started on high-dose steroids. For completion of workup, a bone marrow biopsy was performed which revealed adequate cellular marrow for age (about 50\%) with maturing trilineage hematopoiesis. On day 18 of hospitalization, she received her first dose of chemotherapy regimen with brentuximab, cyclophosphamide, doxorubicin, and steroids (B-CHP). A granulocyte-colony-stimulating factor was administered post chemotherapy.

\section{Follow-up and outcomes}

Chemotherapy treatment was well tolerated, with the improvement of her breathing, lymphadenopathy, energy level, and appetite within a week of her first cycle. She was discharged in 

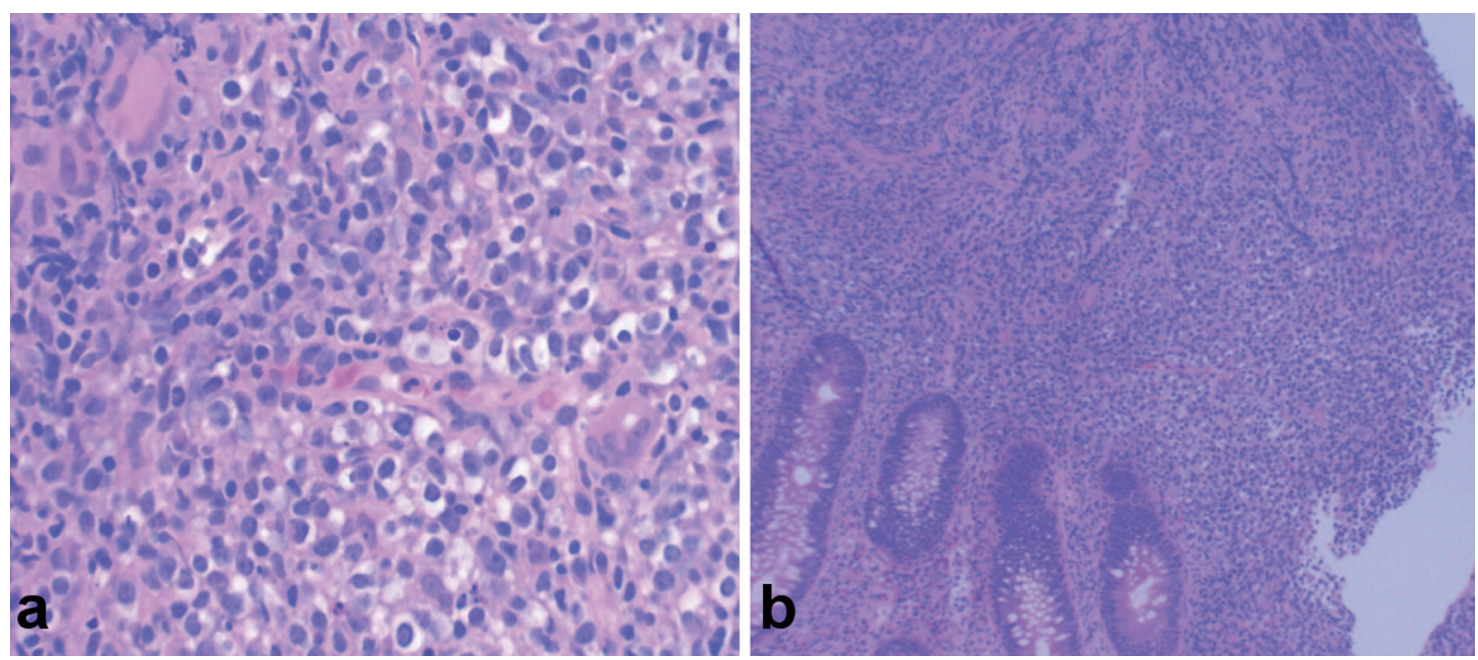

Figure 2. (a, b) Hematoxylin and eosin (H\&E) stain showing colonic mucosa with atypical proliferation of atypical lymphocytes, consistent with angioimmunoblastic T-cell lymphoma (AITL).

a stable condition and has remained oncologically stable with subsequent chemotherapy cycles.

\section{Discussion}

AITL is a type of non-Hodgkin lymphoma and a subset of peripheral $\mathrm{T}$ cell lymphoma that develops from the abnormal formation of $\mathrm{T}$ cells [1]. Although, about $33.3 \%$ of patients diagnosed with AITL can have both abnormal B/T cells present, making it challenging to diagnose [1, 2]. It has a reported incidence of $0.00005 \%$, a median survival of $<3$ years, usually between ages $60-70$, and is responsible for $<3 \%$ of nonHodgkin lymphoma cases [1-4]. Our patient is a quinquage- narian, who had an extensive workup for lymphadenopathy while in Canada, which was inconclusive. Due to an inconclusive diagnosis, her symptoms were thought to be only potential post-COVID-19 complications [5].

AITL features a wide spectrum of clinical symptoms, pathologic manifestations, and distinct molecular characteristics. It usually presents as an advanced stage and is often misdiagnosed, leading to its aggressive course and dismal outcome [4]. As a result of overlapping or confusing symptoms between a variety of benign and malignant lymphoid proliferations, the diagnosis of AITL is rather complex and therefore should include the differential diagnoses to rule out infections, autoimmune disorders, drug reactions, and other lymphoid neoplasms $[4,6]$. Substantially, many clinical features of AITL
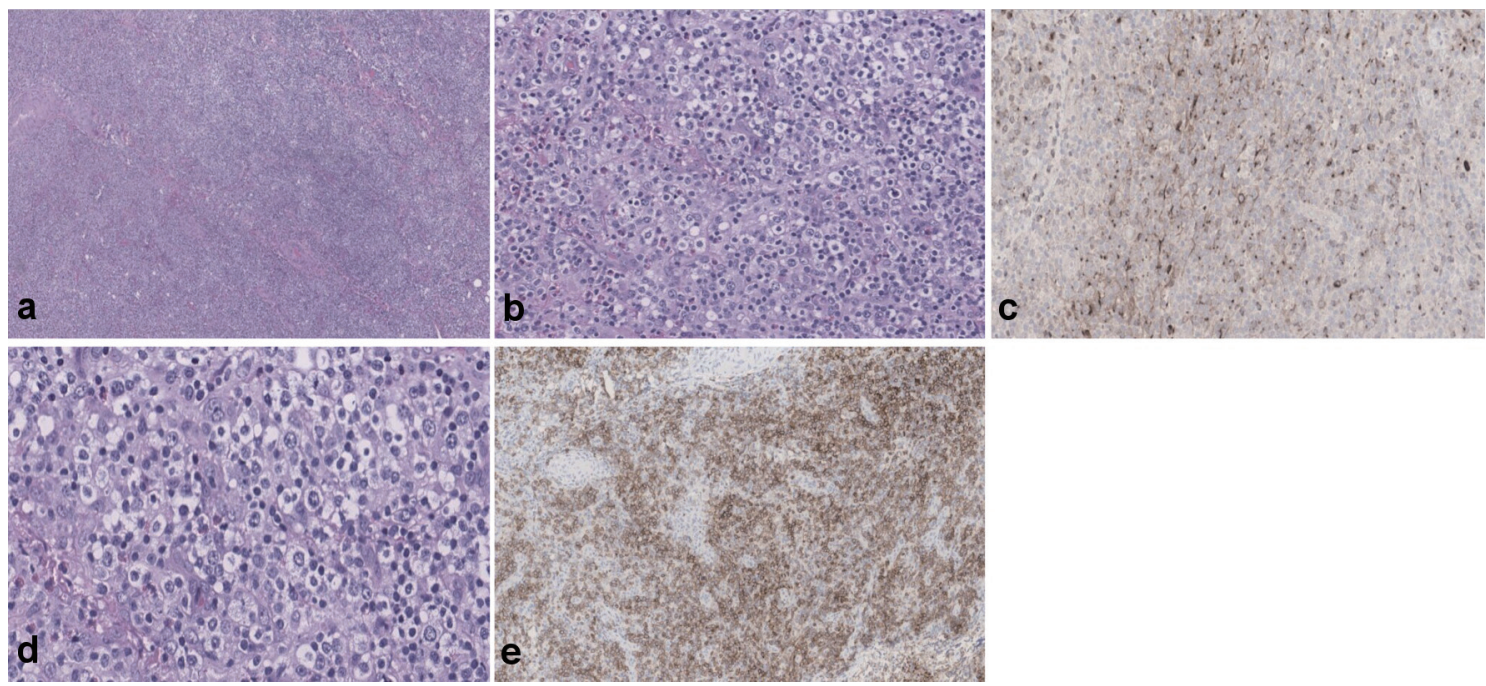

Figure 3. (a-c) Showing portions of lymphoid tissue with effacement of normal architecture by largely diffuse, polymorphic infiltrates of small- to medium-sized lymphocytes (frequently grouped showing clear cytoplasm), scattered large, transformed cells, many eosinophils and plasma cells. Background prominent vasculature, composed of arborizing high endothelial venules is evident. Large, atypical lymphocytes are positive for ICOS and CXCL-13 (d, e). ICOS: inducible T-cell co-stimulator; CXCL-13: chemokine (CXC motif) ligand 13. 
such as skin rash, lymphadenopathy, fever, hemolytic anemia, presence of circulating immune complexes, and rheumatoid factor are more related to infections and immune dysfunction than to tumor growth $[4,7]$. Our patient denied any fever, rash, night sweats, or weight loss. Laboratory tests were negative for SARS-CoV-2 infection, human immunodeficiency virus, hepatitis, tuberculosis, and EBV, ruling out infection. Imaging tests showed diffuse lymphadenopathy raising suspicion for neoplasm.

The lymph node is the main site of involvement for AITL and the gold standard for the diagnosis of the disease is excisional lymph node biopsy [8]. Excisional biopsy is necessary as the architecture of the lymph nodes is effaced in AITL and so helps in morphological diagnosis. A core biopsy is often performed to save time and invasive procedures; however, the sample is usually insufficient to provide an accurate diagnosis as seen in our case [8]. Our patient underwent a core needle biopsy of the axillary lymph node with no definitive diagnosis reached. While in Canada, she was lost to follow-up, thereby prolonging her symptoms, delaying diagnosis, and appropriate management. An excisional biopsy of the left posterior cervical lymph node was performed in our institution and pathology results showed typical features of AITL. Additionally, immunophenotyping is considered a useful method to achieve a confirmed differential diagnosis of AITL $[9,10]$. EBV is positive in $>80 \%$ of cases with AITL, but unlike our patient, EBV was negative. We can argue that her history with SARS-CoV-2 virus infection prior to her initial presenting symptoms in Canada, may have been a trigger for this rare disease [10]. The most common treatment is chemotherapy using cyclophosphamide, doxorubicin, vincristine prednisone (CHOP) regimen for 21 days, followed by autologous stem cell transplantation $[7,8,11]$. The first-line treatment with anthracycline-based chemotherapy has shown complete remission of AITL in $61 \%$ cases, with a 5 -year survival rate of $32 \%[1,12]$. Patients with abnormal B-cells can have rituximab added to the medication regimen [12]. Anti-CD30 antibody, brentuximab vedotin is approved by the Food and Drug Administration (FDA) for the treatment of $\mathrm{CD} 0^{+}$lymphoproliferative disorders including AITL [13]. Recently, results from the ECHELON-2 trial showed that during the treatment of patients $\left(\mathrm{CD} 30^{+}\right)$with peripheral T-cell lymphoma, combination therapy of brentuximab vedotin, cyclophosphamide, doxorubicin, and prednisone $(\mathrm{A}+\mathrm{CHP})$ resulted in a higher median progression-free survival (PFS) of 48.2 months as compared to 20.8 months in the CHOP group alone $(\mathrm{P}=0.011)$ [14]. Our patient is currently on the $\mathrm{A}+\mathrm{CHP}$ therapy regimen and is oncologically stable.

\section{Learning points}

This case report illustrates the diagnostic challenge associated with AITL. Although our patient was evaluated for lymphadenopathy in Canada, she was not consistently followed through. Many times, lymphoma can be hard to catch on the initial biopsy result, so a dedicated effort to follow the patient for months should be made to ensure that the patient does not get lost to follow-up. In addition, an excisional lymph node biopsy is superior to a core biopsy in the diagnosis of this rare disease.

\section{Acknowledgments}

None to declare.

\section{Financial Disclosure}

None to declare.

\section{Conflict of Interest}

None to declare.

\section{Informed Consent}

Informed consent was obtained from the patient before the presentation of this manuscript.

\section{Author Contributions}

Each author has been individually involved in and has made substantial contributions to conceptions and designs, acquisition of data, analysis, interpretation of data, drafting, and editing the manuscript. Ndausung Udongwo contributed to the designs, acquisition of data, analysis, interpretation of data, drafting, and editing of the manuscript; Nusha Fareen contributed to the acquisition of data, drafting, and editing of the manuscript, Hayley Fried contributed to the designs, interpretation of data, drafting, and editing of the manuscript; Jennifer Nessim contributed to the drafting and interpretation of data; LakshmiRehka Narra contributed to the drafting and editing of the manuscript; Dhairya Gor contributed to the drafting and editing of the manuscript; Varsha Gupta contributed to the designs, analysis, and editing of the manuscript; Narmeen Farooq contributed to the analysis and editing of the manuscript; Saira Chaughtai contributed to the designs, analysis, interpretation of data, and editing of the manuscript.

\section{Data Availability}

The authors declare that data supporting the findings of this study are available within the article.

\section{References}

1. Federico M, Rudiger T, Bellei M, Nathwani BN, Luminari S, Coiffier B, Harris NL, et al. Clinicopathologic characteristics of angioimmunoblastic T-cell lymphoma: analysis of the international peripheral T-cell lymphoma project. J Clin Oncol. 2013;31(2):240-246.

2. Morton LM, Wang SS, Devesa SS, Hartge P, Weisenburger DD, Linet MS. Lymphoma incidence patterns by 
WHO subtype in the United States, 1992-2001. Blood. 2006;107(1):265-276.

3. A clinical evaluation of the International Lymphoma Study Group classification of non-Hodgkin's lymphoma. The Non-Hodgkin's Lymphoma Classification Project. Blood. 1997;89(11):3909-3918.

4. Mourad N, Mounier N, Briere J, Raffoux E, Delmer A, Feller A, Meijer CJ, et al. Clinical, biologic, and pathologic features in 157 patients with angioimmunoblastic T-cell lymphoma treated within the Groupe d'Etude des Lymphomes de l'Adulte (GELA) trials. Blood. 2008;111(9):4463-4470.

5. Taweesedt PT, Surani S. Mediastinal lymphadenopathy in COVID-19: A review of literature. World J Clin Cases. 2021;9(12):2703-2710.

6. Xie Y, Jaffe ES. How I diagnose angioimmunoblastic Tcell lymphoma. Am J Clin Pathol. 2021;156(1):1-14.

7. Toure SA, Seck M, Diallo AB, Niang EHD, Keita M, Dabo MF, Faye BF, et al. Diagnosis dilemma of angioimmunoblastic T-cell lymphoma in tuberculosis endemic region. Case Rep Hematol. 2020;2020:8824843.

8. Sachsida-Colombo E, Barbosa Mariano LC, Bastos FQ, Rassi AB, Lage LA, Barreto A, Siqueira S, et al. A difficult case of angioimmunoblastic T-cell lymphoma to diagnose. Rev Bras Hematol Hemoter. 2016;38(1):82-85.

9. Rudiger T, Weisenburger DD, Anderson JR, Armitage JO, Diebold J, MacLennan KA, Nathwani BN, et al. Peripheral T-cell lymphoma (excluding anaplastic large-cell lymphoma): results from the Non-Hodgkin's Lymphoma Classification Project. Ann Oncol. 2002;13(1):140-149.

10. Kanderi T, Goel S, Shrimanker I, Nookala VK, Singh P. Angioimmunoblastic T-cell Lymphoma: An Unusual Case in an Octogenarian. Cureus. 2020;12(2):e6956.

11. Lunning MA, Vose JM. Angioimmunoblastic T-cell lymphoma: the many-faced lymphoma. Blood. 2017; 129(9):1095-1102.

12. Mosalpuria K, Bociek RG, Vose JM. Angioimmunoblastic T-cell lymphoma management. Semin Hematol. 2014;51(1):52-58.

13. Izykowska K, Rassek K, Korsak D, Przybylski GK. Novel targeted therapies of T cell lymphomas. J Hematol Oncol. 2020;13(1):176.

14. Horwitz S, O'Connor OA, Pro B, Illidge T, Fanale M, Advani R, Bartlett NL, et al. Brentuximab vedotin with chemotherapy for CD30-positive peripheral T-cell lymphoma (ECHELON-2): a global, double-blind, randomised, phase 3 trial. Lancet. 2019;393(10168):229240. 